\title{
LINE SEGMENTS ON THE BOUNDARY OF THE NUMERICAL RANGES OF SOME TRIDIAGONAL MATRICES*
}

\author{
ILYA M. SPITKOVSKY ${ }^{\dagger}$ AND CLAIRE MARIE THOMAS ${ }^{\ddagger}$
}

\begin{abstract}
Tridiagonal matrices are considered for which the main diagonal consists of zeroes, the sup-diagonal of all ones, and the entries on the sub-diagonal form a geometric progression. The criterion for the numerical range of such matrices to have line segments on its boundary is established, and the number and orientation of these segments is described.
\end{abstract}

Key words. Numerical range, Tridiagonal matrices, Flat portions.

AMS subject classifications. 15A60.

1. Introduction. Let $M_{n}(\mathbb{C})$ stand for the set of all $n$-by- $n$ matrices with their entries in the field $\mathbb{C}$ of complex numbers. The numerical range (also called the field of values, or the Hausdorff set) of $A \in M_{n}(\mathbb{C})$ is defined as

$$
F(A)=\{\langle A x, x\rangle:\|x\|=1\},
$$

where of course $\langle\cdot, \cdot\rangle$ and $\|\cdot\|$ are the standard scalar product and the norm on $\mathbb{C}^{n}$, respectively. It is well known that $F(A)$ is a convex (the Toeplitz-Hausdorff theorem) compact subset of $\mathbb{C}$ containing the spectrum $\sigma(A)$ of $A$, and thus the convex hull of the latter: $F(A) \supseteq \operatorname{conv} \sigma(A)$. For normal matrices in fact $F(A)=\operatorname{conv} \sigma(A)$, so $F(A)$ is a polygon, and its boundary $\partial F(A)$ consists exclusively of line segments, i.e. "flat portions", and corner points. On the other hand, for a $2 \times 2$ non-normal matrix $A, F(A)$ is an elliptical disk with the foci at the eigenvalues of $A$ (the elliptical range theorem), and the boundary is smooth, with positive curvature throughout.

Starting with $n=3$, however, flat portions of $\partial F(A)$ may exist for not normal, and even unitarily irreducible, $A \in M_{n}(\mathbb{C})$. The possible number of such portions for

\footnotetext{
*Received by the editors on May 20, 2015. Accepted for publication on October 4, 2015. Handling Editor: Panayiotis Psarrakos. The results are partially based on the Capstone project of the second named author under the supervision of the first named author. The latter was also supported in part by the Plumeri Award for Faculty Excellence from the College of William and Mary and by Faculty Research funding from the Division of Science and Mathematics, New York University Abu Dhabi.

${ }^{\dagger}$ Division of Science and Mathematics, New York University Abu Dhabi (NYUAD), Saadiyat Island, P.O. Box 129188 Abu Dhabi, UAE, and Department of Mathematics, College of William and Mary, Williamsburg, VA 23187, USA (ilya@math.wm.edu, ims2@nyu.edu, imspitkovsky@gmail.com).

$\ddagger_{\text {New }}$ York University Abu Dhabi (NYUAD), Saadiyat Island, P.O. Box 129188 Abu Dhabi, UAE (cmt401@nyu.edu, clairemt93@gmail.com).
} 
$n \geq 3$ does not exceed $n(n-1) / 2$ [4, and this bound is sharp if $n=3$ but not for larger values of $n$. More specifically, the sharp upper bound is 4 if $n=4$ [1, Theorem 37], 6 if $n=5$ [7, Lemma 2.2], and not known for $n>5$. A detailed constructive description of the flat portions was obtained in [1 for tridiagonal matrices, that is, when

$$
A=\left[\begin{array}{ccccc}
a_{1} & b_{1} & 0 & \ldots & 0 \\
c_{1} & a_{2} & b_{2} & \ddots & \vdots \\
0 & c_{2} & a_{3} & \ddots & 0 \\
\vdots & \ddots & \ddots & \ddots & b_{n-1} \\
0 & \ldots & 0 & c_{n-1} & a_{n}
\end{array}\right] .
$$

Note that all $A \in M_{n}(\mathbb{C})$ with $n \leq 4$ are tridiagonalizable, that is, unitarily similar to tridiagonal ones. This is a tautology for $n \leq 2$, an easy exercise for $n=3$, and a non-trivial result from [10] for $n=4$. For $n \geq 5$, not all matrices are tridiagonalizable; moreover, the non-tridiagonalizable ones form a dense, second-category subset of $M_{n}(\mathbb{C})[6]$. A concrete example of a non-tridiagonalizable $A \in M_{5}(\mathbb{C})$ can be found in 9 .

In this paper, we concentrate on matrices of the form (1.1) where, in addition,

$$
a_{1}=a_{2}=\cdots=a_{n}(:=a) \text { and }\left\{b_{j}, c_{j}\right\}=\left\{1, z^{j}\right\}, j=1, \ldots, n-1,
$$

for some fixed $z \in \mathbb{C}$. Note that by [2, Lemma 3.1], the numerical range of the matrix (1.1) does not change if the elements of any pair $b_{j}, c_{j}$ of its off diagonal entries are flipped. So, instead of (1.2) we may without loss of generality suppose that $A=a I+A_{n, z}$, where

$$
A_{n, z}=\left[\begin{array}{ccccc}
0 & 1 & 0 & \ldots & 0 \\
z & 0 & 1 & \ddots & \vdots \\
0 & z^{2} & 0 & \ddots & 0 \\
\vdots & \ddots & \ddots & \ddots & 1 \\
0 & \ldots & 0 & z^{n-1} & 0
\end{array}\right] .
$$

Furthermore, for such $A, F(A)=F(n, z)+a$, where we follow [5] in abbreviating $F\left(A_{n, z}\right)$ to $F(n, z)$ for simplicity of notation. So, instead of (1.1)-1.2), we may simply consider matrices of the form (1.3).

By methods different from those of [1], it was established in [3, Theorems 7 and 8] that for all $n \geq 5$ the set $F(n,-1)$ has four flat portions on its boundary. An explanation based on [1] was offered in [5], where the case of arbitrary $z \in \mathbb{C}$ for small matrices $(n \leq 5)$ was also tackled. Here we lift the size restriction. 
2. Auxiliary results. For convenience of reference, we state here several results on tridiagonal matrices which are either known or easily follow from such. Propositions 1 and 2 below are, respectively [2, Lemma 5.1] and [1, Corollary 7].

Proposition 1. A tridiagonal matrix (1.1) is normal if and only if $\left|b_{j}\right|=\left|c_{j}\right|$ for all $j=1, \ldots, n-1, \arg b_{j}+\arg c_{j}$ does not depend on $j$ for all contiguous $j$ such that $b_{j} \neq 0$ (equivalently: $c_{j} \neq 0$ ), and $2 \arg \left(a_{j+1}-a_{j}\right)=\arg b_{j}+\arg c_{j}$ whenever $a_{j} \neq a_{j+1}$ and $b_{j}, c_{j} \neq 0$.

From here it immediately follows:

Corollary 1. Any principal submatrix of a normal tridiagonal matrix is also normal.

As in 11, we will say that a tridiagonal matrix (1.1) is proper if for each $j=$ $1, \ldots, n-1$ at least one of the off diagonal entries $b_{j}, c_{j}$ is different from zero. Of course, for normal proper tridiagonal matrices all $b_{j}$ and $c_{j}$ are different from zero, $j=1, \ldots, n-1$.

Proposition 2. All eigenvalues of a normal proper tridiagonal matrix are simple, and all eigenvectors have non-zero fist and last entries.

For any square matrix $B$ we will denote by $B\left[l_{1}, \ldots, l_{k}\right]$ its principal $k$-by- $k$ submatrix located in the rows and columns numbered $l_{1}, \ldots, l_{k}$.

Proposition 3. The spectra of a normal proper tridiagonal $A \in M_{n}(\mathbb{C})$ and its principle submatrix $A[1, \ldots, n-1]$ are disjoint.

Proof. Suppose $A$ and $A[1, \ldots, n-1]$ do have an eigenvalue in common. Without loss of generality, passing from $A$ to $A-\lambda I$, we may also suppose that this eigenvalue is zero. Being normal by Corollary $1, A[1, \ldots, n-1]$ is unitarily similar to a diagonal matrix $\operatorname{diag}\left[0, \lambda_{2}, \ldots, \lambda_{n-1}\right]$. So, for an appropriately chosen unitary $U \in M_{n-1}(\mathbb{C})$ we have

$$
\left[\begin{array}{cc}
U^{*} & 0 \\
0 & 1
\end{array}\right] A\left[\begin{array}{cc}
U & 0 \\
0 & 1
\end{array}\right]=\left[\begin{array}{cccc|c}
0 & & & & b_{n-1} \bar{u} \\
& \lambda_{2} & & & \\
& & \ddots & & \vdots \\
& & \lambda_{n-1} & * \\
\hline c_{n-1} u & * & \cdots & * & a_{n}
\end{array}\right],
$$

where $u$ is the lower left element of $U$. Consequently,

$$
\operatorname{det}(A)=-b_{n-1} c_{n-1}|u|^{2} \lambda_{2} \cdots \lambda_{n-1} .
$$

Since $\left|b_{n-1}\right|=\left|a_{n-1}\right|$ by Proposition 1 and $\lambda_{2}, \ldots, \lambda_{n-1} \neq 0$ by Proposition 2 , from $\operatorname{det}(A)=0$ it follows that $u=0$. In particular, the first column of the matrix in the 
right hand side of (2.1) is equal to zero. But then (2.1) implies that the eigenvector of $A$ corresponding to its zero eigenvalue is the first column of $U$ augmented by zero. This, however, contradicts the pattern of the non-zero entries of eigenvectors of normal proper tridiagonal matrices, as stated in Proposition 2, $\mathrm{c}$

Of course, a similar statement holds for the principle submatrix $A[2, \ldots, n]$. On the other hand, a simple example of a 3-by-3 matrix $B$ with zero in all four corner positions shows that both $B$ and $B[1,3]$ are singular, while $B$ can be normal, and even hermitian. So, the condition that the $(n-1)$-by- $(n-1)$ submatrix is obtained by deleting either the first or the last row and column is essential.

However, a version of Proposition 3 holds for arbitrary principle submatrices of $A$, provided that we restrict our attention to extreme eigenvalues, that is, the vertices of $F(A)$.

COROllary 2. Let $A$ be a normal proper tridiagonal matrix, and $B$ its arbitrary principal submatrix of a smaller size. Then the vertices of $F(A)$ do not lie in the numerical range of $B$ (and thus are not its eigenvalues).

Proof. Suppose $\lambda \in F(B)$ is a vertex of $F(A)$. Since $F(B) \subset F(A)$, it then has to be also a vertex of $F(B)$ and, moreover, of the numerical range of any principal submatrix $C$ of $A$ containing $B$, and thus $\lambda \in \sigma(C)$. Let us choose $C=A[1, \ldots, n-1]$ if $B$ does not contain the last row and column of $A$. This leads to a contradiction with Proposition 3. The case of $B$ not containing the first row and column of $A$ can be treated similarly.

It remains to consider the case of $B$ being a principle submatrix of $C=A[1, \ldots, k$, $k+1, \ldots, n]$ for some $(1<) k(<n)$. Observe that then $C$ is a block diagonal matrix with the blocks $A[1, \ldots, k-1]$ and $A[k+1, \ldots, n]$. Consequently, $\lambda$ is an eigenvalue of at least one of these blocks. Relabeling this block by $B$, we arrive at the situation already considered.

For our purposes, we need only the version of Corollary 2 for hermitian $A$, in which case it simply means that the eigenvalues of any (strictly smaller) principle submatrix $B$ of $A$ lie strictly between the extreme eigenvalues of $A$. This is a small, but important for us, addition to the interlacing theorem, see example on pg. 185 of [8] for the statement of the latter.

Finally, we state the criterion for flat portions to exist on the boundary of the numerical range for matrices (1.1). This is a slightly reworded [1, Theorem 10].

TheOREM 1. Let $A$ be a proper tridiagonal matrix of the form (1.1). Then $\partial F(A)$ contains a line segment at an angle $\theta$ from the positive $x$-axis if and only if 
(i) The set set $J=\left\{j: b_{j}=e^{2 i \theta} \overline{c_{j}}\right\}$ is non-empty: $J=\left\{j_{1}, \ldots, j_{m-1}\right\}$ for some $\left(j_{0}=0<\right) j_{1}<\cdots<j_{m-1}\left(<j_{m}=n\right), m>1 ;$

(ii) The minimal or maximal eigenvalue $\mu$ of $\operatorname{Im}\left(e^{-i \theta} A\right)$ is attained by at least two of its diagonal blocks $\operatorname{Im}\left(e^{-i \theta} A_{k}\right)$, where

$$
A_{k}=A\left[j_{k-1}+1, \ldots, j_{k}\right], \quad k=1, \ldots, m .
$$

(iii) Among the blocks $\operatorname{Im}\left(e^{-i \theta} A_{k}\right)$ satisfying (ii), either there are two adjacent ones, or for their unit eigenvectors $x_{k}$ corresponding to the eigenvalue $\mu$ the values $\operatorname{Re}\left(e^{-i \theta}\left\langle A_{k} x_{k}, x_{k}\right\rangle\right)$ are not all the same.

Note that the matrices $\operatorname{Im}\left(e^{-i \theta} A_{k}\right)$ are proper tridiagonal and hermitian. So, according to Proposition 2, their eigenvalues are simple and the eigenvectors have non-zero first and last entries. This justifies the simplification made in the statement of Theorem [1 compared to [1, Theorem 10], where the simplicity of $\mu$ and nonzero requirement on the first/last entries of $x_{l}, x_{l+1}$ in case of adjacent $\operatorname{Im}\left(e^{-i \theta} A_{l}\right)$, $\operatorname{Im}\left(e^{-i \theta} A_{l+1}\right)$ were explicitly mentioned.

3. Main result. As was already observed in [5], condition (i) of Theorem 1 implies that flat portions on the boundary of $F(n, z)$ are possible only if $|z|=1$. Besides, matrices $A_{n, 1}$ are hermitian, with $F(n, 1)=\left[-2 \cos \frac{\pi}{n+1}, 2 \cos \frac{\pi}{n+1}\right]$. So, we will from now on suppose $z$ unimodular and different from one. The following lemma will play a key role.

LEMma 1. Let in (1.3) $z$ be unimodular and let $\omega$ be a square root of $z$. Then for any $m, n$ and $k \leq \min \{m, n-m\}$, the matrices

$$
B_{1}=\operatorname{Im}\left(\omega^{-m} A_{n, z}[m-k+1, \ldots, m]\right) \text { and } B_{2}=\operatorname{Im}\left(\omega^{-m} A_{n, z}[m+1, \ldots, m+k]\right)
$$

have the same spectra.

Proof. Both $B_{1}$ and $B_{2}$ are tridiagonal hermitian $k$-by- $k$ matrices with zero main diagonal. Such matrices are completely determined by their first sup-diagonal, that is, the $(k-1)$-string of their $(i, i+1)$ entries, $i=1, \ldots, k-1$. So, for brevity of notation we will operate with these strings in place of the matrices per se. This string for $B_{1}$ is

$$
\frac{1}{2 i}\left[\omega^{-m}-\omega^{-m+2 k-2}, \omega^{-m}-\omega^{-m+2 k-4}, \ldots, \omega^{-m}-\omega^{-m+2}\right] .
$$

Denoting by $Z\left(=Z^{T}\right) \in M_{k}(C)$ the permutational matrix corresponding to the order reversing permutation $\sigma=\left(\begin{array}{cccc}1 & 2 & \cdots & k \\ k & k-1 & \cdots & 1\end{array}\right)$, observe that $Z B_{1}^{T} Z$ has the supdiagonal string

$$
\frac{1}{2 i}\left[\omega^{-m}-\omega^{-m+2}, \omega^{-m}-\omega^{-m+4}, \ldots, \omega^{-m}-\omega^{-m+2 k-2}\right] .
$$


In its turn, the unitary similarity via $V=\operatorname{diag}\left[v_{1}, \ldots, v_{k}\right]$, where $v_{j}=(-1)^{j} \omega^{-j(j-1)}$, yields the matrix $V^{*}\left(Z B_{1}^{T} Z\right) V$ with the sup-diagonal

$$
\frac{1}{2 i}\left[\omega^{-m}-\omega^{-m-2}, \omega^{-m}-\omega^{-m-4}, \ldots, \omega^{-m}-\omega^{-m-2 k+2}\right],
$$

which coincides with the sup-diagonal of $B_{2}$. Thus, $B_{1}$ and $B_{2}$ can be transformed one into another via unitary similarities and transpositions, and so have the same spectra.

In the statement of our main result, an important role will be played by the order of $z$ as a root of unity. We will denote this order by $p$, meaning that $p=\infty$ if $z$ is not a root, and that $z, \ldots, z^{p-1} \neq 1=z^{p}$ otherwise.

Note that the tridiagonal matrices $B_{1}$ and $B_{2}$ from Lemma 1 are proper provided that $k<p$. Combining the results of Lemma 1 and Corollary 2 we thus obtain:

COROLlary 3. Let in (1.3) $z$ be a root of unity of order $p$, and let $\omega$ be a square root of $z$. Then for any $m, n$ and $k_{1}, k_{2} \leq \min \{m, n-m, p-1\}, k_{1} \neq k_{2}$, the matrices $B_{1}=\operatorname{Im}\left(\omega^{-m} A_{n, z}\left[m-k_{1}+1, \ldots, m\right]\right)$ and $B_{2}=\operatorname{Im}\left(\omega^{-m} A_{n, z}\left[m+1, \ldots, m+k_{2}\right]\right)$ have different maximal eigenvalues.

A similar statement of course holds for the minimal eigenvalues.

Proof. Indeed, if say $k_{1}<k_{2}$, then by Lemma 1 the spectrum of $B_{1}$ coincides with that of the upper left $k_{1}$-by- $k_{1}$ principle submatrix $B_{3}$ of $B_{2}$. In its turn, $\sigma\left(B_{3}\right)$ lies strictly between the minimal and maximal eigenvalues of $B_{2}$, because the latter is a proper tridiagonal matrix (since $k_{2}<p$ ), and thus Corollary 2 is applicable.

Finally, recall that the set $F(n, z)$ is centrally symmetric [5, Lemma 1], and so the flat portions on its boundary, if any, come in parallel pairs.

THEOREM 2. Let in (1.3) $z$ be a root of unity of order $p>1$. Then the number $N(n, z)$ of parallel pairs of the flat portions on the boundary of $F(n, z)$ and their orientation are determined by the following rules:

$$
N(n, z)= \begin{cases}0 & \text { if } n<2 p \text { is odd, } \\ 1 & \text { if } n \leq 2 p \text { is even, } \\ \min \{p, n-2 p+1\} & \text { if } n>2 p .\end{cases}
$$

The angle formed by these flat portions with the positive $x$-axis is $\theta_{j}=(j \arg z) / 2$, $j=0, \ldots, \min \{p-1, n-2 p\}$ if $n>2 p$, and $\theta=(n \arg z) / 4$ if $n \leq 2 p$ is even.

Proof. Condition (i) of Theorem 1 holds if and only if $\theta$ attains one of the $n-1$ values

$$
\theta_{k}=(k \arg z) / 2, \quad k=1, \ldots, n-1 .
$$


If $n \leq 2 p$, then for $\theta=\theta_{k}$ in the notation of the same Theorem 1 we have $J=\{k\}$, $m=2$, and $A_{1}=A[1, \ldots, k], A_{2}=A[k+1, \ldots, n]$. By Corollary [3, the matrices $\operatorname{Im}\left(e^{-i \theta_{k}} A_{1}\right)$ and $\operatorname{Im}\left(e^{-i \theta_{k}} A_{2}\right)$ will have different maximal and minimal eigenvalues whenever their sizes are different, that is, when $k \neq n-k$. On the other hand, if their sizes happen to coincide, they will have the same spectra due to Lemma 1. Consequently, condition (ii) of Theorem 1 is satisfied if and only if $n$ is even and $k=n / 2$; the respective (unique) value of $\theta$ is given by (3.3) with $k=n / 2$ thus equaling $(n \arg z) / 4$. Since the blocks $A_{1}$ and $A_{2}$ are adjacent, condition (iii) is then satisfied automatically. This proves the first two lines of (3.2) and verifies the value of $\theta$ corresponding to the second of them.

Let now $n>2 p$, implying in particular that $p$ is finite and thus $z$ is indeed a root of unity. Then only $p$ of the $\theta_{k}$ given by (3.3) define different directions, and we may choose any $p$ pairwise different $\bmod p$ of them. It is notation-wise convenient to relabel them in (3.3) by $k=0, \ldots, p-1$.

For $k=n-2 p+1, \ldots, p-1$ (which is a non-vacuous set only if $n<3 p-1$ ) we will have $m=3, J=\{k, k+p\}$, and $\operatorname{Im}\left(e^{-i \theta_{k}} A\right)$ splits into three proper tridiagonal blocks, the middle of them being $p$-by- $p$, and two others having strictly smaller size. By Corollary 3, the minimal and maximal eigenvalues of $\operatorname{Im}\left(e^{-i \theta_{k}} A\right)$ are attained by its middle block only. Thus, condition (ii) of Theorem 1 is not satisfied for the angles $\theta_{k}$ in the considered range.

Consider now the remaining values of $k=0, \ldots, p-1$, that is, $k=0, \ldots, \min \{p-$ $1, n-2 p\}$. The matrix $\operatorname{Im}\left(e^{-i \theta_{k}} A\right)$ then splits into at least two contiguous identical $p$-by- $p$ blocks, preceded and/or succeeded by a block of strictly smaller size. The maximal and minimal eigenvalues of $\operatorname{Im}\left(e^{-i \theta_{k}} A\right)$ are therefore attained by its $p$-by- $p$ blocks, and so conditions (ii) and (iii) of Theorem 1 are met.

COROLlary 4. Let in (1.3) $z$ be a root of unity of order $p>1$ while $n \geq 3 p-1$. Then there are exactly $p$ parallel pairs of the flat portions on $\partial F(n, z)$, and the angles $j \pi / p, j=0, \ldots, p-1$, do not depend on the particular choice of $z$.

Note that for $n<3 p-1$ the number of flat portions depends on the particular value of $n$ and their orientation depends on the specific choice of $z$.

\section{Examples.}

ExAmple 1. Let $p=2$, that is, $z=-1$. According to Theorem 2 there are no flat portions on $\partial F(3,-1)$, one pair of horizontal flat portions on $\partial F(4,-1)$, and two pairs (one horizontal, and one vertical) on $\partial F(n,-1)$ for $n \geq 5$. This is in complete agreement with Theorem 8 of $\underline{3}$. Note that formally $\partial F(2,-1)$ also should contain two flat portions; what happens though is that $F(2,-1)$ is a vertical line segment, that is, the two flat portions in this case degenerate into one. 
ExAmple 2. Let $n=4$. Then $n \leq 2 p$ unless $z=1$. In agreement with [5, Theorem 4], we see from our Theorem 2 that for all unimodular $z \neq 1$ there is one pair of flat portions on $\partial F(4, z)$, forming the $\operatorname{angle} \arg z$ with the positive $x$-axis.

ExAmple 3. Let $n=5$ and $z \neq \pm 1$. Then $p \geq 3$, and so $n<2 p$. According to Theorem 2, there are no flat portions on the boundary of $F(5, z)$ - the fact established earlier in [5, Theorem 5].

ExAmple 4 . Let $n=6$ and $z \neq \pm 1$. Then $p \geq 3$, and so $n<2 p$. According to Theorem 2, there is exactly one pair of parallel flat portions, at the angle of $3(\arg z) / 2$ with the positive $x$-axis. The following figures illustrate this point, for $z=e^{2 \pi i / 3}$ and $z=-e^{3 \pi i / 7}$.

Fig. 4.1. $A\left(6, e^{2 \pi i / 3}\right)$ (left) and $A\left(6,-e^{3 \pi i / 7}\right)$ (right).

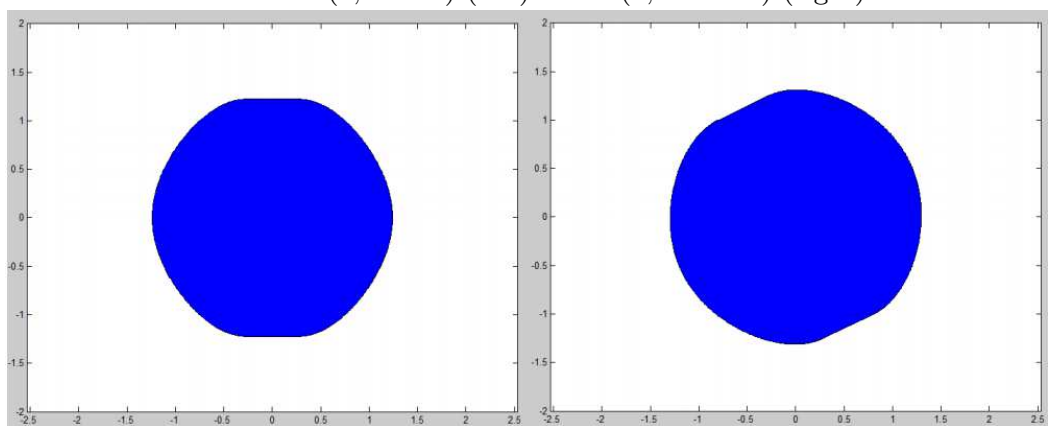

ExAmple 5. Let $n>6$ and $p=3$. By Theorem 2, in this case $N(n, z)=$ $\min \{3, n-5\}$, and so there will be 2 pairs of parallel flat portions if $n=7$ and 3 such pairs otherwise. Below are the corresponding figures for $z=e^{2 \pi i / 3}$ and $n=7,8,9,10$ and 13 .

FIG. 4.2. $A\left(7, e^{2 \pi i / 3}\right)$.

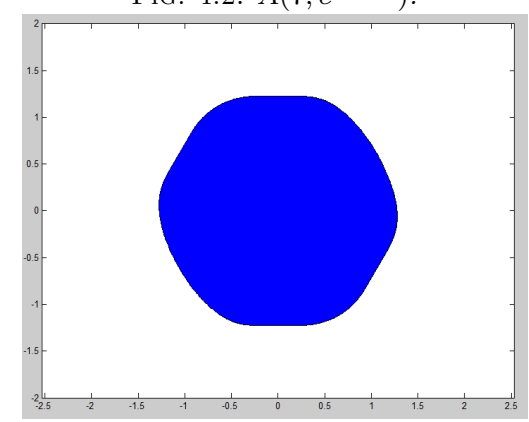


Electronic Journal of Linear Algebra ISSN 1081-3810

A publication of the International Linear Algebra Society

Volume 30, pp. 693-703, October 2015

Fig. 4.3. $A\left(8, e^{2 \pi i / 3}\right)$.

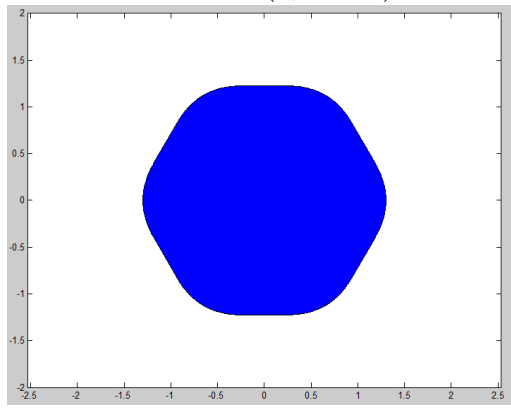

FIG. 4.4. $A\left(9, e^{2 \pi i / 3}\right)$

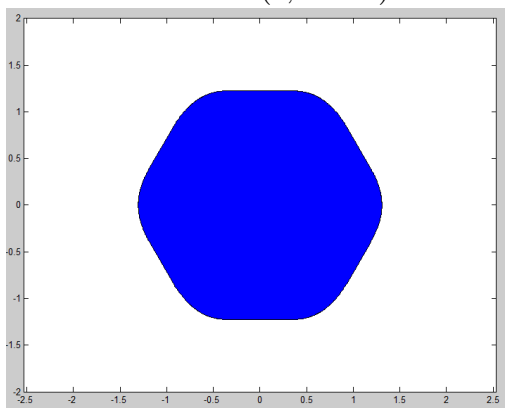

FIG. 4.5. $A\left(10, e^{2 \pi i / 3}\right)$.

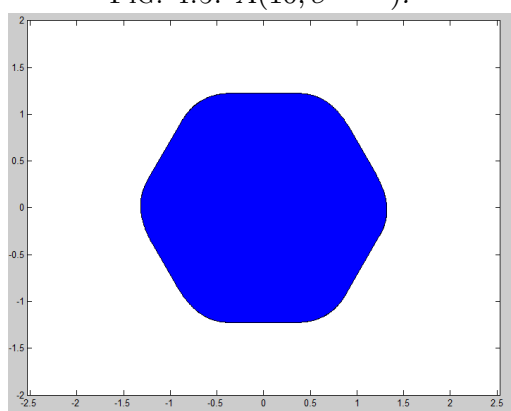


Electronic Journal of Linear Algebra ISSN 1081-3810

A publication of the International Linear Algebra Society

Volume 30, pp. 693-703, October 2015

Fig. 4.6. $A\left(13, e^{2 \pi i / 3}\right)$.

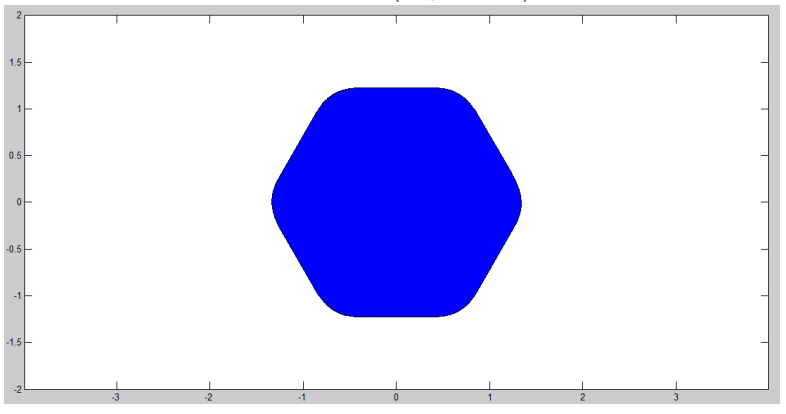

ExAmple 6. Finally, the figures below illustrate Corollary 4 .

FIG. 4.7. $A(13, i)$.

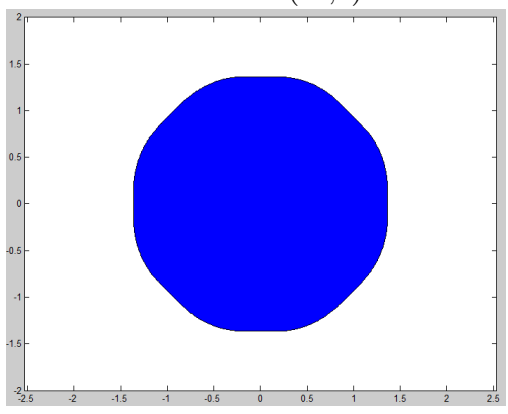

FIG. 4.8. $A\left(15,-e^{\pi i / 5}\right)$.

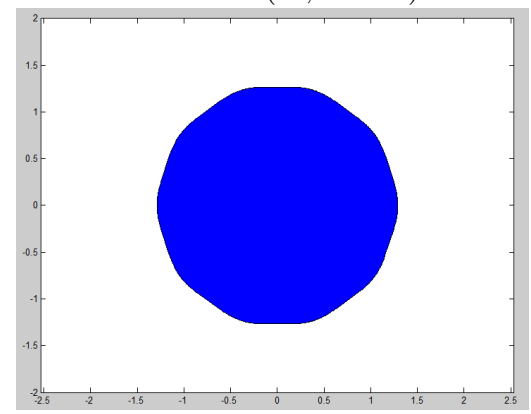




\section{REFERENCES}

[1] E. Brown and I. Spitkovsky. On flat portions on the boundary of the numerical range. Linear Algebra Appl., 390:75-109, 2004.

[2] E. Brown and I. Spitkovsky. On matrices with elliptical numerical ranges. Linear Multilinear Algebra, 52:177-193, 2004.

[3] M.T. Chien and H. Nakazato. The numerical range of a tridiagonal operator. J. Math. Anal. Appl., 373(1):297-304, 2011.

[4] M.T. Chien and H. Nakazato. Flat portions on the boundary of the numerical ranges of certain Toeplitz matrices. Linear and Multilinear Algebra, 56:143-162, 2008.

[5] R.T. Chien and I.M. Spitkovsky. On the numerical ranges of some tridiagonal matrices. Linear Algebra Appl., 470:228-240, 2015.

[6] C.K. Fong and P.Y. Wu. Band-diagonal operators. Linear Algebra Appl., 248:185-204, 1996.

[7] H.-L. Gau and P.Y. Wu. Line segments and elliptic arcs on the boundary of a numerical range. Linear Multilinear Algebra, 56(1-2):131-142, 2008.

[8] R.A. Horn and C.R. Johnson. Matrix Analysis. Cambridge University Press, New York, 1985.

[9] T. Leake, B. Lins, and I.M. Spitkovsky. Pre-images of boundary points of the numerical range. Oper. Matrices, 8:699-724, 2014.

[10] V. Pati. Unitary tridiagonalization in $M(4, \mathbb{C})$. Proc. Indian Acad. Sci. Math. Sci., 111(4):381397, 2001. 